\title{
COASSOCIATIVE GRAMMAR, PERIODIC ORBITS, AND QUANTUM RANDOM WALK OVER $\mathbb{Z}$
}

\author{
PHILIPPE LEROUX
}

Received 13 January 2005 and in revised form 20 September 2005

Inspired by a work of Joni and Rota, we show that the combinatorics generated by a quantisation of the Bernoulli random walk over $\mathbb{Z}$ can be described from a coassociative coalgebra. Relationships between this coalgebra and the set of periodic orbits of the classical chaotic system $x \mapsto 2 x \bmod 1, x \in[0,1]$, are also given.

\section{Introduction and notation}

Motivated by the success of classical random walks and chaotic dynamical systems, we study the quantisation of the random walk over $\mathbb{Z}$ and its relationships with a classical chaotic system $x \mapsto 2 x \bmod 1, x \in[0,1]$. In the physics literature, quantum random walks have been studied, for instance, by Ambainis et al. [1] and Konno et al. [8, 9]. In [8], Konno shows that the quantum random walk over $\mathbb{Z}$ called the Hadamard random walk generates a particular combinatorics. In [6], Joni and Rota showed that some combinatorics can be recovered from coproducts of coassociative coalgebras. Therefore, is it possible to create a coassociative coalgebra which recovers the combinatorics generated by the Hadamard random walk? We start in Section 2 with briefly recalling a new formalism, inspired by weighted directed graph theory. In Section 3, we present a mathematical framework for studying the Hadamard random walk over $\mathbb{Z}$. In Section 4, we construct a coassociative coalgebra based on results on graphs developed in Section 2. We show that the combinatorics generated by the Hadamard random walk over $\mathbb{Z}$ can be recovered from this coalgebra. In Section 5, we present the notion of quantum graphs developed in [15] and point out a relation between a quantum graph, the classical Bernoulli random walk, the Hadamard random walk, and the periodic orbits of the classical chaotic system $x \mapsto 2 x \bmod 1$ with $x \in[0,1]$. Briefly speaking, we show how to relate periodic orbits of this classical chaotic system to polynomials describing a quantisation of the Bernoulli walk.

\section{2. $L$-coalgebras}

In the sequel, $k$ denotes a field. A directed graph $G$ is a quadruple [16] $\left(G_{0}, G_{1}, s, t\right)$, where $G_{0}$ and $G_{1}$ are two countable sets, respectively, called the vertex set and the arrow set. 
No multiple arrows are allowed in this work. The two mappings $s, t: G_{1} \rightarrow G_{0}$ are, respectively, called source and terminus. A vertex $v \in G_{0}$ is a source (resp., a sink) if $t^{-1}(\{v\})$ (resp., $\left.s^{-1}(\{v\})\right)$ is empty. A graph $G$ is said to be locally-finite (resp., row-finite) if $t^{-1}(\{v\})$ is finite (resp., $s^{-1}(\{v\})$ is finite). Let us fix a vertex $v \in G_{0}$. Define the set $F_{v}:=\{a \in$ $\left.G_{1}, s(a)=v\right\}$. A weight associated with the vertex $v$ is a mapping $w_{v}: F_{v} \rightarrow k$. A directed graph equipped with a family of weights $w:=\left(w_{v}\right)_{v \in G_{0}}$ is called a weighted directed graph. In the sequel, directed graphs will be supposed locally-finite and row-finite. Let us introduce particular coalgebras named $L$-coalgebras, introduced in [12] and developed in $[13,14]$, and explain why this notion is interesting. An $L$-coalgebra $(L, \Delta, \tilde{\Delta})$ over a field $k$ is a $k$-vector space composed of a right part $(L, \Delta)$, where $\Delta: L \rightarrow L^{\otimes 2}$ is called the right coproduct, and a left part $(L, \tilde{\Delta})$, where $\tilde{\Delta}: L \rightarrow L^{\otimes 2}$ is called the left coproduct, such that the coassociativity breaking equation, $(\tilde{\Delta} \otimes \mathrm{id}) \Delta=(\mathrm{id} \otimes \Delta) \tilde{\Delta}$, is verified. If $\Delta=\tilde{\Delta}$, the $L$ coalgebra is said to be degenerate. An $L$-coalgebra may have two counits, the right counit $\epsilon: L \rightarrow k$, verifying (id $\otimes \epsilon) \Delta=\mathrm{id}$, and the left counit $\tilde{\epsilon}: L \rightarrow k$, verifying $(\tilde{\epsilon} \otimes \mathrm{id}) \tilde{\Delta}=\mathrm{id}$. An $L$-coalgebra is said to be coassociative if its two coproducts are coassociative. In this case, the equation, $(\tilde{\Delta} \otimes \mathrm{id}) \Delta=(\mathrm{id} \otimes \Delta) \tilde{\Delta}$, is called the entanglement equation and we will say that its right part $(L, \Delta)$ is entangled to its left part $(L, \tilde{\Delta})$. Denote by $\tau$ the transposition mapping, that is, $L^{\otimes 2} \stackrel{\tau}{\rightarrow} L^{\otimes 2}$ such that $\tau(x \otimes y)=y \otimes x$, for all $x, y \in L$. The $L$-coalgebra $L$ is said to be $L$-cocommutative if $\Delta=\tau \tilde{\Delta}$. Let $G=\left(G_{0}, G_{1}, s, t\right)$ be a directed graph equipped with a family of weights $\left(w_{v}\right)_{v \in G_{0}}$. Let us consider the free $k$-vector space $k G_{0}$. The set $G_{1}$ is then viewed as a subset of $\left(k G_{0}\right)^{\otimes 2}$ by identifying $a \in G_{1}$ with $s(a) \otimes t(a)$. The mappings source and terminus are then linear mappings still called source and terminus $s, t:\left(k G_{0}\right)^{\otimes 2} \rightarrow k G_{0}$, such that $s(u \otimes v)=u$ and $t(u \otimes v)=v$, for all $u, v \in G_{0}$. The family of weights is given by $\left(w_{v}: F_{v} \rightarrow k\right)_{v \in G_{0}}$. Let $v \in G_{0}$. Define the right coproduct $\Delta_{M}: k G_{0} \rightarrow\left(k G_{0}\right)^{\otimes 2}$, such that $\Delta_{M}(v):=\sum_{i: a_{i} \in F_{v}} w_{v}\left(a_{i}\right) v \otimes t\left(a_{i}\right)$, and the left coproduct $\tilde{\Delta}_{M}: k G_{0} \rightarrow\left(k G_{0}\right)^{\otimes 2}$, such that $\tilde{\Delta}_{M}(v):=\sum_{i: a_{i} \in P_{v}} w_{s}\left(a_{i}\right)\left(a_{i}\right) s\left(a_{i}\right) \otimes v$, where $P_{v}$ is the set $\left\{a \in G_{1}, t(a)=v\right\}$. With these definitions the $k$-vector space $k G_{0}$ is an $L$-coalgebra called a (finite) Markov L-coalgebra since its coproducts $\Delta_{M}$ and $\tilde{\Delta}_{M}$ verify the coassociativity breaking equation $\left(\tilde{\Delta}_{M} \otimes \mathrm{id}\right) \Delta_{M}=\left(\mathrm{id} \otimes \Delta_{M}\right) \tilde{\Delta}_{M}$. In addition, this particular coalgebra is called finite since for all $v \in G_{0}$, the sets $F_{v}$ and $P_{v}$ are finite and the coproducts have the form $\Delta_{M}(v):=v \otimes \cdots$ and $\tilde{\Delta}_{M}(v):=\cdots \otimes v$, see [12]. Consider the Markov L-coalgebra just described and associate with each tensor product $\lambda u \otimes v$, where $\lambda \in k$ and $u, v \in G_{0}$, appearing in the definition of the coproducts, a directed arrow $u \stackrel{\lambda}{\rightarrow} v$. The weighted directed graph so obtained, called the geometric support of this $L$-coalgebra, is up to a graph isomorphism, the directed graph we started with. Therefore, general $L$-coalgebras generalise the notion of weighted directed graphs. If $(L, \Delta, \tilde{\Delta})$ is an $L$-coalgebra spanned as a $k$-vector space by a set $L_{0}$, then its geometric support $\operatorname{Gr}(L)$ is a directed graph with vertex set $\operatorname{Gr}(L)_{0}=L_{0}$ and with arrow set $\operatorname{Gr}(L)_{1}$, the set of tensor products $u \otimes v$, with $u, v \in L_{0}$, appearing in the definition of the coproducts of $L$. As a coassociative coalgebra is a particular $L$-coalgebra, we naturally construct its directed graph. We draw attention to the fact that a directed graph can be the geometric support of different $L$-coalgebras.

Example 2.1. The directed graph shown in Figure 2.1 is the geometric support associated with the degenerate $L$-coalgebra or coassociative coalgebra $\mathscr{E}$, spanned by the basis $a$, $b, c$, and $d$, as a $k$-vector space, and described by the coproduct $\Delta(a)=a \otimes a+b \otimes c$, 


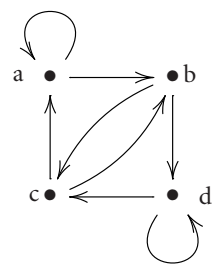

Figure 2.1. The graph $\operatorname{Gr}(\mathscr{E})$.

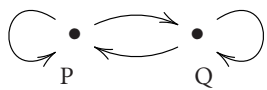

Figure 2.2. The (2,1)-De Bruijn graph.

$\Delta(b)=a \otimes b+b \otimes d, \Delta(c)=d \otimes c+c \otimes a, \Delta(d)=d \otimes d+c \otimes b$ and the geometric support of the finite Markov $L$-coalgebra, spanned by the basis $a, b, c$, and $d$, as a $k$-vector space, and described by the right coproduct $\Delta_{M}(a)=a \otimes(a+b), \Delta_{M}(b)=b \otimes(c+d), \Delta_{M}(c)=$ $c \otimes(a+b), \Delta_{M}(d)=d \otimes(c+d)$ and the left coproduct $\tilde{\Delta}_{M}(a)=(a+c) \otimes a, \tilde{\Delta}_{M}(b)=(a+$ $c) \otimes b, \tilde{\Delta}_{M}(c)=(b+d) \otimes c, \tilde{\Delta}_{M}(d)=(b+d) \otimes d$.

Example 2.2 (the (2,1)-De Bruijn graph). The graph shown in Figure 2.2, also called the $(2,1)$-De Bruijn graph, is the geometric support of a Markov $L$-coalgebra, spanned by the basis $P$ and $Q$. The (coassociative) coproducts are $\Delta(P)=P \otimes P+P \otimes Q$ and $\Delta(Q)=$ $Q \otimes P+Q \otimes Q, \tilde{\Delta}(P)=Q \otimes P+P \otimes P$ and $\tilde{\Delta}(Q)=P \otimes Q+Q \otimes Q$.

Let $G$ be a finite Markov $L$-coalgebra. If the family of weights used for describing right and left coproducts takes values in $\mathbb{R}_{+}$and if the right counit $\epsilon: v \mapsto 1$ exists, then the geometric support associated with $G$ is a directed graph equipped with a family of probability vectors. In addition, to enlarge the coassociative coalgebra setting, this algebraic formalism takes also into account the description of weighted paths on a given directed graph. We recall that the sequence $\Delta_{1} \equiv \Delta, \Delta_{2}=\mathrm{id} \otimes \Delta, \Delta_{3}=\mathrm{id} \otimes \mathrm{id} \otimes \Delta, \ldots$ generates all possible weighted paths starting at any vertex. Similarly, the sequence of powers of $\tilde{\Delta}$ generates all the possible weighted paths arriving at a given vertex. We end this part on graph theory by recalling the definition of the line-extension. The line-extension of a directed graph $G:=\left(G_{0}, G_{1}, s, t\right)$ with a denumerable vertex set $G_{0}$ and a denumerable arrow set $G_{1} \subseteq G_{0} \times G_{0}$ is the directed graph with vertex set $G_{1}$ and arrow set $G_{2} \subseteq G_{1} \times G_{1}$ defined by $(v, w) \in G_{1} \times G_{1}$ belonging to $G_{2}$ if and only if $t(v)=s(w)$. This directed graph, called the line-directed graph of $G$, is denoted by $E(G)$. The line-extension of the $(2,1)$-De Bruijn graph is $\operatorname{Gr}(\mathscr{E})$.

\section{Quantum random walk over $\mathbb{Z}$}

In the physics literature, quantum random walks were studied, for instance, by Ambainis et al. [1] and Konno et al. [8, 9]. Here, we propose a mathematical framework for the 


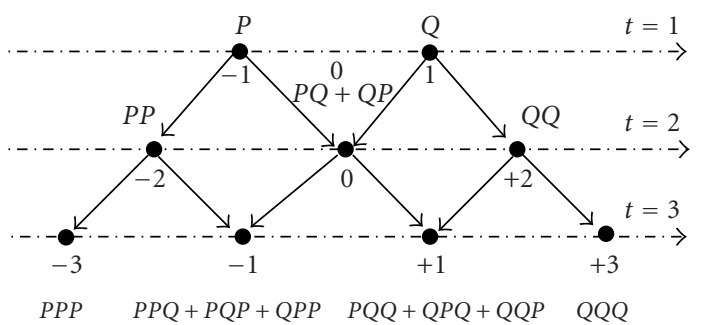

Figure 3.1. Combinatorics from the quantum random walk over $\mathbb{Z}$, up to $t=3$.

quantum random walk over $\mathbb{Z}$ and show that the combinatorics (we keep the notation of [8]) of this walk can be recovered by using the coproduct of $\mathscr{E}$. Let $\mathscr{H}$ be a separable Hilbert space of infinite dimension with $(|n\rangle)_{n \in \mathbb{Z}}$ as an orthonormal basis. Consider the trivial tensor bundle $\mathscr{H} \otimes M_{2}(\mathbb{C})$. Fix a unitary matrix $U$ and consider the operators $P$ and $Q$, such that $U=P+Q$, with

$$
P=\left(\begin{array}{ll}
\alpha & \beta \\
0 & 0
\end{array}\right), \quad Q=\left(\begin{array}{ll}
0 & 0 \\
\gamma & \delta
\end{array}\right)
$$

They verify the following algebraic relations [8]: $P^{2}=\alpha P, Q^{2}=\delta Q, P Q P=\beta \gamma P, Q P Q=$ $\beta \gamma Q$.

Remark 3.1. Suppose $\alpha \delta \neq 0$. Consider $e_{1}:=(1 / \alpha) P$ and $e_{2}:=(1 / \delta) Q$. We get $e_{1}^{2}=e_{1}$, $e_{2}^{2}=e_{2}, e_{1} e_{2} e_{1}=\lambda e_{1}$, and $e_{2} e_{1} e_{2}=\lambda e_{2}$, where $\lambda:=\gamma \beta / \delta \alpha$, that is, the algebra generated by $e_{1}, e_{2}$ is a Jones algebra [5].

Consider the algebra $\mathbb{C}\langle P, Q\rangle$, that is, the noncommutative polynomials in $P$ and $Q$, and denote by $\mathscr{D}_{-}, \mathscr{D}_{+}$the diffusion operators, that is, the linear maps

$$
\left.\left.\mathscr{D}_{-}, \mathscr{D}_{+}: \mathscr{H}_{\mathbb{C}} \otimes P, Q\right\rangle \longrightarrow \mathscr{H}_{\mathbb{C}} \otimes P, Q\right\rangle,
$$

which are defined for all $k \in \mathbb{Z}$ and for all discrete time $n \in \mathbb{Z}$ such that

$$
\mathscr{D}_{-}\left(|k+1\rangle \otimes \Xi_{[k+1 ; n]}\right)=|k\rangle \otimes \Xi_{[k+1 ; n]} P, \quad \mathscr{D}_{+}\left(|k-1\rangle \otimes \Xi_{[k-1 ; n]}\right)=|k\rangle \otimes \Xi_{[k-1 ; n]} Q
$$

where $\Xi_{[0 ; 0]}=\mathrm{id}, \Xi_{[-1 ; 1]}=P, \Xi_{[+1 ; 1]}=Q$, and so on. The dynamics is defined by

$$
|k\rangle \otimes \Xi_{[k ; n+1]}:=\mathscr{D}_{-}\left(|k+1\rangle \otimes \Xi_{[k+1 ; n]}\right)+\mathscr{D}_{+}\left(|k-1\rangle \otimes \Xi_{[k-1 ; n]}\right) .
$$


That is shown in Figure 3.1 (graphically, the Hilbert space $\mathscr{H}$ will be represented by the usual representation of $\mathbb{Z}$, a vertex $n \in \mathbb{Z}$ meaning $|n\rangle \in \mathscr{H}$ ).

Example 3.2. We give here the nonzero polynomials $\Xi_{[k ; n+1]}$ at time $t=0, \ldots, 4$. At time $t=0$, we get, by convention, $\Xi_{[0 ; 0]}=\mathrm{id}$. At time $t=1, \Xi_{[-1 ; 1]}=P, \Xi_{[+1 ; 1]}=Q$. At time $t=2, \Xi_{[-2 ; 2]}=P^{2}, \Xi_{[0 ; 2]}=P Q+Q P$, and $\Xi_{[+2 ; 2]}=Q^{2}$. At time $t=3, \Xi_{[-3 ; 3]}=P^{3}, \Xi_{[-1 ; 3]}=$ $Q P^{2}+P Q P+P^{2} Q, \Xi_{[+1 ; 3]}=P Q^{2}+Q P Q+Q^{2} P$, and $\Xi_{[3 ; 3]}=Q^{3}$. At time $t=4, \Xi_{[-4 ; 4]}=$ $P^{4}, \Xi_{[-2 ; 4]}=Q P^{3}+P Q P^{2}+P^{2} Q P+P^{3} Q, \Xi_{[0 ; 4]}=P^{2} Q^{2}+P Q P Q+P Q^{2} P+Q^{2} P^{2}+Q P Q P+$ $Q P^{2} Q, \Xi_{[+2 ; 4]}=P Q^{3}+Q P Q^{2}+Q^{2} P Q+Q^{3} P$, and $\Xi_{[+4 ; 4]}=Q^{4}$

Denote by $S\left(\mathbb{C}^{2}\right)$ the set of vectors $\psi$ of $S\left(\mathbb{C}^{2}\right)$ such that $\psi^{\dagger} \psi=1$. The quantum random walk over $\mathbb{Z}$ from a state $\psi \in S\left(\mathbb{C}^{2}\right)$ is defined by the initial condition $\Psi_{\text {space }=0 \text {, time }=0}:=$ $|0\rangle \otimes \psi$. At time $n$, this state will spread and the probability amplitude at position $k$ described by $|k\rangle$ will be $\Psi_{k, n}:=|k\rangle \otimes \Xi_{[k ; n+1]} \psi$ ( since $P^{\dagger} P+Q^{\dagger} Q=I$, the norm of the initial state is preserved). We have an action from the bundle $\mathscr{H}_{\mathbb{Z}} \otimes \mathbb{C}\langle P, Q\rangle$ on $\mathbb{C}^{2}$ described by

$$
\mathscr{R} W: \mathscr{H}_{\mathbb{Z}} \otimes \mathbb{C}\langle P, Q\rangle \times \mathbb{C}^{2} \longrightarrow \mathscr{H}_{\mathbb{Z}} \otimes \mathbb{C}^{2}, \quad\left(|k\rangle \otimes \Xi_{[k ; n+1]}, \psi\right) \longmapsto|k\rangle \otimes \Xi_{[k ; n+1]} \psi .
$$

The total state is $\Psi_{\text {total }}^{n}:=\sum_{k} \Psi_{k, n}$.

Remark 3.3. For all $x \in \mathbb{C}\langle P, Q\rangle$, we define the right polynomial multiplication $R_{x}$ : $\mathbb{C}\langle P, Q\rangle \rightarrow \mathbb{C}\langle P, Q\rangle, y \mapsto y x$; we have $\left[\mathscr{D}_{+}, \mathscr{D}_{-}\right]=\mathrm{id} \otimes R_{[Q, P]}$.

\section{Noncommutative polynomials and the reading of periodic orbits of $\operatorname{Gr}(\mathscr{E})$}

Remark 4.1. From now on, we forget the algebraic relation between $P$ and $Q$. These monomials will be treaded simply as noncommutative symbols with no relation between them. Observe also that any monomial in $\mathbb{C}\langle P, Q\rangle$ is in one-to-one correspondence with a path of the $(2,1)$-De Bruijn graph. For instance, the monomial PPPQP corresponds to the path shown in Figure 4.1 of the (2,1)-De Bruijn graph.

The aim of this section is to recover the polynomials $\Xi_{[k ; n]}$, involved in the quantum random walk, from the periodic orbits of $\operatorname{Gr}(\mathscr{E})$. We will show that the periodic orbits of this directed graph allow us to recover the combinatorics generated by the quantum random walk over $\mathbb{Z}$ and that this combinatorics is generated by the coassociative coproduct of $\mathscr{E}$. Recall that this coproduct is defined by $\Delta(a)=a \otimes a+b \otimes c, \Delta(b)=a \otimes b+b \otimes d$, $\Delta(c)=d \otimes c+c \otimes a, \Delta(d)=d \otimes d+c \otimes b$. However, this directed graph can be also embedded into its natural Markov $L$-coalgebra. Recall that by definition the right coproduct $\Delta_{M}$ verifies $\Delta_{M}(a)=a \otimes(a+b), \Delta_{M}(b)=b \otimes(c+d), \Delta_{M}(c)=c \otimes(a+b), \Delta_{M}(d)=$ $d \otimes(c+d)$.

Definition 4.2 (path space). Denote by $\mathscr{F}_{n}$ the free $k$-vector space spanned by all the monomials $x_{1} \otimes \cdots \otimes x_{n}$, where for all $1 \leq i \leq n, x_{i}$ stands for $a, b, c, d$ and such that $x_{1} \cdots x_{n}$ represents a path of length $n$ of the graph $\operatorname{Gr}(\mathscr{E})$. For instance, $\mathscr{F}_{0}:=k\langle a, b, c, d\rangle$. The path space of the graph $\operatorname{Gr}(\mathscr{E})$ is called the $k$-vector space $\mathscr{F}_{\mathscr{F}}:=\bigoplus_{n \geq 0} \mathscr{F}_{n}$. 


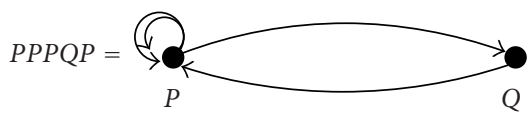

Figure 4.1. Path associated with $P P P Q P$.

Remark 4.3. As described in Section 2, we associate with each tensor product $P \otimes Q$, appearing in the definition of the coproducts, a directed arrow $P \rightarrow Q$, the relationship between the $(2,1)$-De Bruijn graph, whose vertex set is $\{P, Q\}$, and its line-extension is thus given by setting $a:=P \otimes P, b:=P \otimes Q, c:=Q \otimes P$, and $d:=Q \otimes Q$.

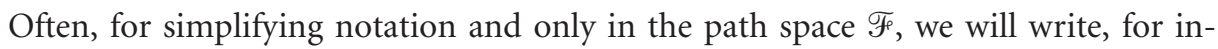
stance, $x y$ instead of $x \otimes y$, where $x, y$ stand for $a, b, c, d$. No confusion is possible since, in the sequel, we forget the algebraic relations between letters. Define now the contraction map.

Definition 4.4 (contraction map). For all $n>1$, the contraction map is the linear map

$\mathscr{C}: \mathscr{F} \longrightarrow \mathbb{C}\langle P, Q\rangle, \quad\left(y_{1} \otimes y_{2}\right) \otimes\left(y_{2} \otimes y_{3}\right) \otimes\left(y_{3} \otimes y_{4}\right) \cdots\left(y_{n-1} \otimes y_{n}\right) \longmapsto y_{1} y_{2} \cdots y_{n}$,

where for all $1 \leq i \leq n, y_{i}$ stands for $P$ and $Q$. For instance, the contraction of $a \otimes b \otimes c:=$ $(P \otimes P) \otimes(P \otimes Q) \otimes(Q \otimes P)$ is equal to $P P Q P$.

Proposition 4.5. Fix a time $t>1$. To any monomial $\Xi$ constructed from $P$ and $Q$ in the algebra $\mathbb{C}\langle P, Q\rangle$, excepted of course $P$ and $Q$, corresponds a unique monomial $\omega$ in $\mathscr{F}$ such that $\mathscr{C}(\omega)=\Xi$.

Proof. Any monomial $\Xi$ constructed from $P$ and $Q$ in the algebra $\mathbb{C}\langle P, Q\rangle$ corresponds to a unique path of the $(2,1)$-De Bruijn graph, that is, a unique path of its line-extension.

Lemma 4.6. If $x$ stands for $a, b, c$ or $d$, the following equalities hold:

$$
\begin{array}{lll}
\mathscr{C}(x \otimes a) P=\mathscr{C}(x \otimes a \otimes a), & \mathscr{C}(x \otimes a) Q=\mathscr{C}(x \otimes a \otimes b), \\
\mathscr{C}(x \otimes b) P=\mathscr{C}(x \otimes b \otimes c), & \mathscr{C}(x \otimes b) Q=\mathscr{C}(x \otimes b \otimes d), \\
\mathscr{C}(x \otimes c) P=\mathscr{C}(x \otimes c \otimes a), & \mathscr{C}(x \otimes c) Q=\mathscr{C}(x \otimes c \otimes b), \\
\mathscr{C}(x \otimes d) P=\mathscr{C}(x \otimes d \otimes c), & \mathscr{C}(x \otimes d) Q=\mathscr{C}(x \otimes d \otimes d) .
\end{array}
$$

Let $x \in\{a, b, c, d\}$. Then $\mathscr{C}(x)(P+Q)=\mathscr{C}\left(\Delta_{M}(x)\right)$.

Proof. The proof comes from simple computations.

Define $\left(\Delta_{M}\right)_{0}=\mathrm{id},\left(\Delta_{M}\right)_{1}:=\Delta_{M},\left(\Delta_{M}\right)_{2}:=\left(\mathrm{id} \otimes \Delta_{M}\right) \Delta_{M}$ and, more generally, for all $n>0,\left(\Delta_{M}\right)_{n}:=(\underbrace{\mathrm{id} \otimes \cdots \otimes \mathrm{id}}_{n-1} \otimes \Delta_{M})\left(\Delta_{M}\right)_{n-1}$, similarly for the coassociative coproduct $\Delta$. 


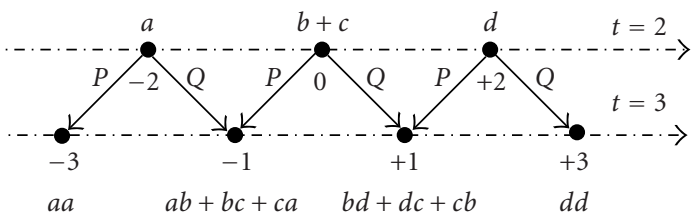

Figure 4.2. The quantum random walk, coded in terms of the path space $\mathscr{F}$.

Proposition 4.7. The following equalities $\Delta_{M}(a+b)=\Delta(a+b)$ and $\Delta_{M}(c+d)=$ $\Delta(c+d)$ hold. Moreover, (id $\otimes \Delta) \Delta_{M}=\left(\mathrm{id} \otimes \Delta_{M}\right) \Delta_{M}$. The equalities (id $\left.\otimes \Delta\right) \Delta(a+b)=$ $\left(\mathrm{id} \otimes \Delta_{M}\right) \Delta_{M}(a+b)$ and $(\mathrm{id} \otimes \Delta) \Delta(c+d)=\left(\mathrm{id} \otimes \Delta_{M}\right) \Delta_{M}(c+d)$ imply that $\left(\Delta_{M}\right)_{n}(a+b+$ $c+d)=(\Delta)_{n}(a+b+c+d)$ for all $n \geq 0$.

Proof. It is straightforward.

Corollary 4.8. Fix $n>1$. Denote $\Sigma_{n}:=\sum_{-n \leq k \leq n} \Xi_{[k, n]}$. Then $\Sigma_{n}=\mathscr{C}\left(\left(\Delta_{M}\right)_{n-2}(a+b+c+\right.$ $d))=\mathscr{C}\left((\Delta)_{n-2}(a+b+c+d)\right)$.

Remark 4.9. Set $n>1$. The polynomial $\Sigma_{n}$ is the sum of all the monomials $\Xi_{[k, n]}$, appearing exactly one time because of Lemma 4.6, generated by the combinatorics of the quantum random walk over $\mathbb{Z}$. This sum can be computed by contraction of all the monomials from $\mathscr{F}$ present at time $n$ and obtained either by applying the operator $\left(\Delta_{M}\right)_{n-2} \cdots\left(\Delta_{M}\right)_{2}$ $\left(\Delta_{M}\right)_{1}$ to $a+b+c+d$ or by applying the operator $\Delta_{n-2} \cdots \Delta_{2} \Delta_{1}$ to $a+b+c+d$.

Here is the beginning of the combinatorics generated by the quantum random walk over $\mathbb{Z}$, viewed from the path space $\mathscr{F}$, as shown in Figure 4.2.

For the moment, we get all the sums $\Sigma_{n}$ of monomials created by the walk. If a monomial is picked up from $\mathscr{F}_{F}$ or from a sum $\Sigma_{n}$, how can we say that it has to belong to such or such vertex? We have to enlarge the definition of $\mathscr{F}$ by defining an index map and an index path space. From now on, we denote by convention $x_{-1,-1}:=a, x_{-1,+1}:=b$, $x_{+1,-1}:=c, x_{+1,+1}:=d$ and observe that a monomial from $\mathscr{F}$ can be always written like $\omega:=x_{i_{1} i_{2}} x_{i_{2} i_{3}} \cdots x_{i_{n-1} i_{n}}$. The index path space $\hat{\mathscr{F}}$ is by definition the space $\mathscr{H} \otimes \mathscr{F}$.

Definition 4.10 (index map). Let $\omega \in \mathscr{F}$, say $\omega:=x_{i_{1} i_{2}} x_{i_{2} i_{3}} \cdots x_{i_{n-1} i_{n}}$. The (linear) index map is defined as ind : $\mathscr{F} \rightarrow \widehat{\mathscr{F}}, \omega \mapsto(|\operatorname{ind}(\omega)\rangle \otimes \omega)$, with ind $(\omega)=\operatorname{ind}\left(x_{i_{1} i_{2}} x_{i_{2} i_{3}} \cdots x_{i_{n-1} i_{n}}\right):=$ $\sum_{k=1}^{n} i_{k}$.

Proposition 4.11. Let $\omega:=x_{i_{1} i_{2}} \cdots x_{i_{n-1} i_{n}} \in \mathscr{F}$. The index ind $(\omega)$ is equal to the number of $Q$ minus the number of $P$ obtained in the contraction of the monomial $\omega$. Therefore, the index map fixes the vertex attributed by the quantum random walk over $\mathbb{Z}$.

Proof. We will proceed by induction. It is true for $n=2$, that is, for $a, b, c, d$. Let $\omega$ be a monomial present at vertex $k$ and at time $t=n>2$. We suppose $\omega=x_{i_{1} i_{2}} \cdots x_{i_{n-1} i_{n}}$ and the index ind $(\omega)=k$ does indicate the number of $Q$ minus the number of $P$ obtained in the contraction of this monomial. At time $t=n+1, \omega \mapsto \omega \otimes x_{i_{n}, i_{n+1}}$. By definition of the quantum random walk, this monomial will be at vertex $k+1$ if $x_{i_{n}, i_{n+1}}$ is equal to $Q$ or $k-1$ if $x_{i_{n}, i_{n+1}}$ is equal to $P$. Now, $\operatorname{ind}\left(\omega \otimes x_{i_{n}, i_{n+1}}\right)=\operatorname{ind}(\omega)+i_{n+1}$. By definition, $i_{n+1}=+1$ for $b$ 


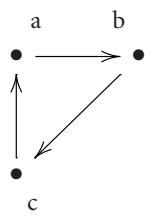

Figure 4.3. Periodic orbit, $\langle a \otimes b \otimes c\rangle$, with pattern of length 3 .

and $d$ which are monomials ending by $Q$ and $i_{n+1}=-1$ for $a$ and $c$ which are monomials ending by $P$.

Example 4.12. For instance, ind $(a)=-2$ and $\mathscr{C}(a)=P^{2}$. Therefore, the monomial $a$ has to be present at time $t=2$. Moreover, its contraction yielding the monomial $P^{2}, a$ is at vertex -2 , as expected.

By using the projector $|k\rangle\langle k| \otimes$ id, we will recover all the monomials $\omega$ of the sum $\Sigma_{n}$ with index ind $(\omega)=k$. The next question is how can we produce all these monomials of $\mathscr{F}_{\mathscr{F}}$ from the notion of periodic orbits of the $(2,1)$-De Bruijn graph.

Definition 4.13 (periodic orbits, pattern). The equivalence relation $\sim$ in $\mathscr{F}$ is defined by saying that $\omega_{1} \sim \omega_{2}$ if and only if $\omega_{1}=x_{i_{1} i_{2}} x_{i_{2} i_{3}} \cdots x_{i_{n-1} i_{1}}$, for some $n$ and there exist $m$, $\tau^{m}\left(\omega_{1}\right)=\omega_{2}$, where $\tau^{m}: \mathscr{F} \rightarrow \mathscr{F}$ is such that $y_{1} \cdots f_{m} \mapsto f_{p-m+1} \cdots f_{p} f_{1} \cdots f_{p-m}$. The set $\mathbb{P O}=\mathscr{F} / \sim$ is the set of periodic orbits of the directed graph $\operatorname{Gr}(\mathscr{E})$. Denote by $\langle\omega\rangle$ the pattern of an equivalence classe associated with $\omega$ and its permutations, that is, $\langle\omega\rangle:=$ $\left\langle x_{i_{1} i_{2}} x_{i_{2} i_{3}} \cdots x_{i_{n-1} i_{1}}\right\rangle$. A periodic orbit is just the graphical representation of the pattern. Often, the two words will be confounded.

Remark 4.14. Fix a time $t>1$. It is straightforward that the length of the pattern of a periodic orbit $\langle\omega\rangle$ present at $t$ denoted by $l(\langle\omega\rangle)$ is equal to $t$.

Example 4.15. We have $a \otimes b \otimes c \sim c \otimes a \otimes b \sim b \otimes c \otimes a$. The equivalent classe is designed by the pattern $\langle a \otimes b \otimes c\rangle$ and the associated periodic orbit is ... abcabcabcabc... as shown in Figure 4.3.

This periodic orbit can be also represented by the pattern $\langle a \otimes b \otimes c \otimes a \otimes b \otimes c\rangle$, that is, we cover two times the triangle. Similarly, we have to enlarge the $k$-vector space of the periodic orbits $\mathbb{P O}$ to keep the notion of vertex attributed by the quantum walk to each periodic orbit. Denote by $\widehat{\mathbb{P O}}:=\mathscr{H} \otimes \mathbb{P} \mathbb{O}$ such a set.

Definition 4.16 (index map in $\mathbb{P O}$ ). Define the (linear) index map Ind : $\mathbb{P} \mathbb{O} \rightarrow \widehat{\mathbb{P O}},\langle\omega\rangle \mapsto$ $|\operatorname{Ind}(\langle\omega\rangle)\rangle \otimes\langle\omega\rangle$ with

$$
\begin{aligned}
\operatorname{Ind}(\langle\omega\rangle) & :=\operatorname{Ind}\left(\left\langle x_{i_{1} i_{2}} x_{i_{2} i_{3}} \cdots x_{i_{n-1} i_{1}}\right\rangle\right) \\
& :=\frac{1}{2}\left(\left(i_{1}+i_{2}\right)+\left(i_{2}+i_{3}\right)+\cdots+\left(i_{n-1}+i_{1}\right)\right)=\sum_{k=1}^{n-1} i_{k} .
\end{aligned}
$$




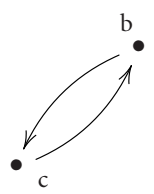

Figure 4.4. Periodic orbit of period $4,\langle b c b c\rangle$, with pattern of length 4 .

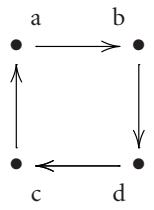

Figure 4.5. Periodic orbit, $\langle a b d c\rangle$, with pattern of length period 4 .

This definition does not depend on the choice of the representative of the equivalent classe. Once we have the definition of periodic orbits, we have to read them to obtain information.

Definition 4.17 (reading map). Let $\langle\omega\rangle:=\left\langle x_{i_{1} i_{2}} x_{i_{2} i_{3}} \cdots x_{i_{n} i_{1}}\right\rangle$ be a periodic orbit. The reading map is denoted by $R: \mathbb{P O} \rightarrow F$ with $x_{i_{1} i_{2}} x_{i_{2} i_{3}} \cdots x_{i_{n} i_{1}} \mapsto \sum_{k=1}^{N} x_{i_{k} i_{k+1}} x_{i_{k+1} i_{k+2}} \cdots x_{i_{n+k-2} i_{k+n-1}}$, the labels being understood modulo $n$. Define $X_{k}:=x_{i_{k} i_{k+1}} x_{i_{k+1} i_{k+2}} \cdots x_{i_{n+k-2} i_{k+n-1}}$ for all $1 \leq$ $k \leq n$, the integer $N$ is equal to $\min \left\{k ; X_{i} \neq X_{j}, 1 \leq i, j \leq k\right\}$. The reading map does not depend on the choice of the representative of the equivalent classe.

Proposition 4.18. Under notation in the definition of the reading map, $X_{N+k \bmod n}=X_{k}$.

Proof. Let us show that $X_{N+1}=X_{1}$. Indeed, if $k \leq N$ is such that $X_{N+1}=X_{k}$, then it is straightforward to show that $X_{N+2-k}=X_{1}$ and $N+1-k=\min \left\{l ; X_{i} \neq X_{j}, 1 \leq i, j \leq l\right\}$. Therefore, we get $N+2-k=N+1$, only possible for $k=1$.

Example 4.19. Consider the periodic orbit $\langle a b c\rangle$. Its index is -1 and its reading yields $a b+b c+c a$. By contraction, we obtain $P P Q+P Q P+Q P P$, which is exactly the polynomial expected at time $t=3$ and at vertex -1 .

Example 4.20. The reading of the periodic orbit $\langle b \otimes c \otimes b \otimes c\rangle$, with a pattern of length 4, as shown in Figure 4.4, yields the monomials $X_{1}=b \otimes c \otimes b, X_{2}=c \otimes b \otimes c, X_{3}=b \otimes$ $c \otimes b$, and $X_{4}=c \otimes b \otimes c$. Therefore, $N=2$ and $R\langle b \otimes c \otimes b \otimes c\rangle:=b \otimes c \otimes b+c \otimes b \otimes c$. By contraction, we obtain $P Q P Q+Q P Q P$. Moreover, its index is 0 .

The reading of the periodic orbit $\langle a \otimes b \otimes d \otimes c\rangle$, with pattern of length 4 , as shown in Figure 4.5, yields $a \otimes b \otimes d+b \otimes d \otimes c+d \otimes c \otimes a+c \otimes a \otimes b$. By contraction, we obtain $P P Q Q+P Q Q P+Q Q P P+Q P P Q$. Its index is 0 .

That is, the sum of these two periodic orbits gives the polynomials expected at time $t=4$ and at vertex 0 . 


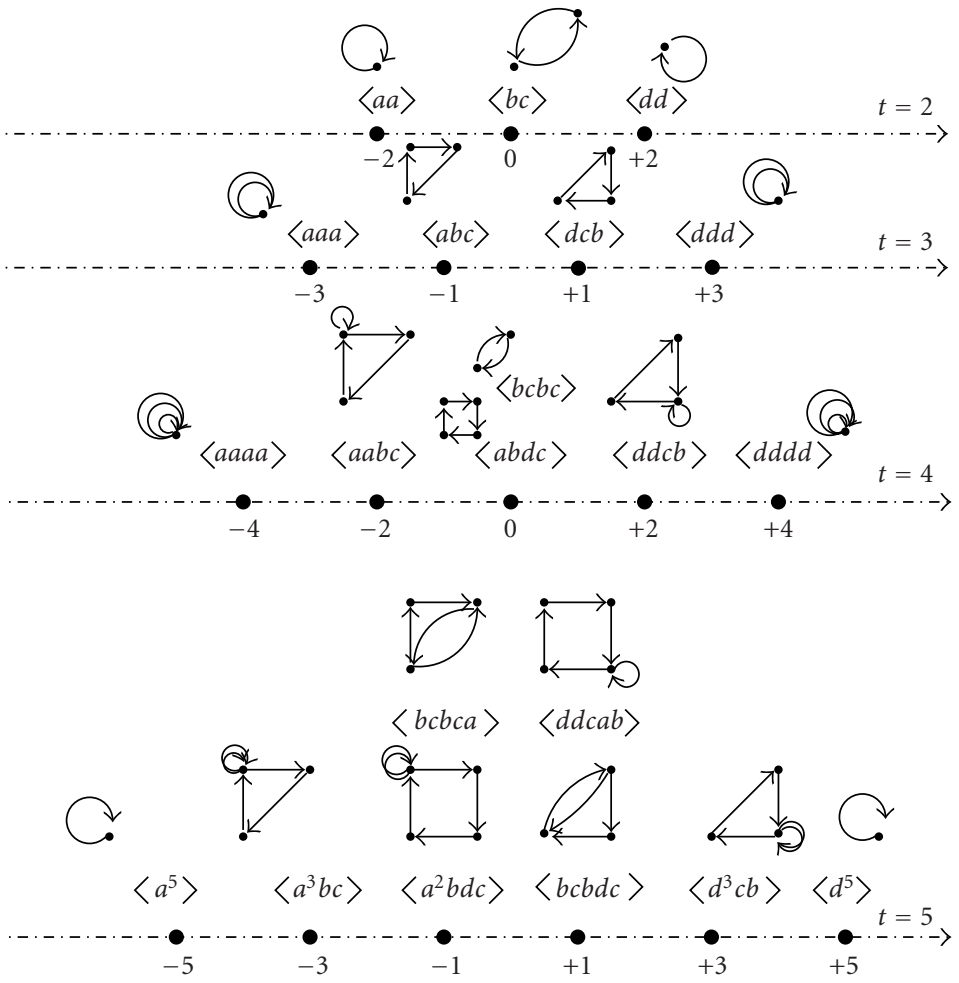

Figure 4.6. Periodic orbits and their associated pictures.

So as to give a minorant of the number of periodic orbits at a given time $n$ at vertex $k$, we have to link the number $k$ to the number $x_{P}$ of $P$ and the number $x_{Q}$ of $Q$. Suppose $k$ positive (as the walk is symetric, we have as many polynomials at vertex $k$ as at vertex $-k$ ). This means that $x_{Q}=x_{P}+k$. As $x_{P}+x_{Q}=n$, we get $x_{P}=(n-k) / 2$. As this solution has to be an integer, this will fix the possibilities of $k$, that is, the possible vertices reached by the quantum random walk at time $n$. Set $\kappa:=(n-k) / 2$.

Proposition 4.21. Set $₫:=(1 / n)(n ! / \kappa !(n-\kappa) !)$. The number of periodic orbits at the vertex $k$ and at time $n$ is greater than or equal to $@$ if this number is an integer and greater than or equal to the integer part of $\omega+1$ if $\emptyset$ is not an integer.

Proof. For a given vertex $k$ and a given time $n$, we have to have $n ! / \kappa !(n-\kappa)$ ! polynomials in $P$ and $Q$ and by definition we know that the reading of these periodic orbits yields at most $n$ different monomials.

In Figure 4.6, we indicate the periodic orbits (of the line-extension) of the $(2,1)$-De Bruijn graph involved in the quantum walk over $\mathbb{Z}$, up to $t=5$.

Proposition 4.22. Let $\langle\omega\rangle:=\left\langle x_{i_{1} i_{2}} x_{i_{2} i_{3}} \cdots x_{i_{n} i_{1}}\right\rangle$ be the pattern of a periodic orbit. Its read-

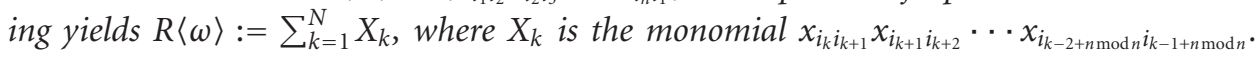


We have $\operatorname{Ind}(\langle\omega\rangle):=\operatorname{ind}\left(X_{k}\right)$, for all $k=1, \ldots, n$, that $i$, the reading map decomposes the periodic orbit into monomials with same index.

Proof. Let $\langle\omega\rangle:=\left\langle x_{i_{1} i_{2}} x_{i_{2} i_{3}} \cdots x_{i_{n} i_{1}}\right\rangle$ be a periodic orbit and $X_{1}, \ldots, X_{N}$ its decomposition under the reading map. We have $s_{1}:=\operatorname{ind}\left(X_{1}\right):=\operatorname{ind}\left(x_{i_{1} i_{2}} x_{i_{2} i_{3}} \cdots x_{i_{n-1} i_{n}}\right)=\sum_{k=1}^{n} i_{k}$, $s_{2}:=\operatorname{ind}\left(X_{2}\right):=\operatorname{ind}\left(x_{i_{2} i_{3}} x_{i_{3} i_{4}} \cdots x_{i_{n-1} i_{n}} x_{i_{n} i_{1}}\right)=\sum_{k=2}^{n} i_{k}+i_{1}$. Thus $s_{1}=s_{2}$. Similarly, $s_{k}:=$ $\operatorname{ind}\left(X_{k}\right):=i_{k}+i_{k+1}+i_{k+2}+\cdots+i_{n}+i_{1}+\cdots+i_{k-1}=s_{1}$. Besides, by definition, $\operatorname{Ind}(\langle\omega\rangle):=$ $(1 / 2)(2 \operatorname{ind}(\omega))$.

Remark 4.23. A periodic orbit with index $k$ will be assigned to the vertex $k$, by applying, for example, the projector $|k\rangle\langle k| \otimes \mathrm{id}: \widehat{\mathbb{P O}} \rightarrow \widehat{\mathbb{P O}}$. Its reading will yield monomials of index $k$. For instance, the reading of the periodic orbit $\langle a b c\rangle$ is $R(\langle a b c\rangle):=a b+b c+c a$. We have $\operatorname{Ind}(\langle a b c\rangle)=-1$ and $\operatorname{ind}(a b)=\operatorname{ind}(c a)=\operatorname{ind}(b c)=-1$.

Definition 4.24 (completion). For a time $t=n$ and for a given vertex $k$, all the polynomials can be recovered from the contraction of monomials of $\mathscr{F}_{\mathscr{F}}$ and having the same index $k$. Suppose we pick up one of the monomials present at this vertex $k$, say $x_{i_{1} i_{2}} x_{i_{2} i_{3}} \cdots x_{i_{n-1} i_{n}}$. The completion Comp maps $x_{i_{1} i_{2}} x_{i_{2} i_{3}} \cdots x_{i_{n-1} i_{n}}$ to $\left\langle x_{i_{1} i_{2}} x_{i_{2} i_{3}} \cdots x_{i_{n-1} i_{n}} x_{i_{n} i_{1}}\right\rangle$, thus it is a map from $\mathscr{F}$ to $\mathbb{P O}$. Similarly, $\widehat{\operatorname{Comp}}: \widehat{\mathscr{F}} \rightarrow \widehat{\mathbb{P O}}$ can be defined by $\widehat{\text { Comp }}:=\mathrm{id} \otimes$ Comp thanks to Proposition 4.25 .

Proposition 4.25. $\operatorname{Ind}\left(\operatorname{Comp}\left(x_{i_{1} i_{2}} x_{i_{2} i_{3}} \cdots x_{i_{n-1} i_{n}}\right)\right)=\operatorname{ind}\left(x_{i_{1} i_{2}} x_{i_{2} i_{3}} \cdots x_{i_{n-1} i_{n}}\right)$.

Proof. It is straightforward.

The reading of the completion of a monomial of $\mathscr{F}$ present at vertex $k$ will yield many monomials of $\mathscr{F}$ present at this vertex. By contraction, we will recover polynomials in $P$ and $Q$ present at vertex $k$. As all these polynomials in $P$ and $Q$ are coded bijectively through monomials of $\mathscr{F}$ present at the vertex $k$, the completion of all these monomials will form all the necessary periodic orbits whose reading will yield all the monomials of $\mathscr{F}_{\mathscr{F}}$ present at $k$. For the time being, we started with the polynomial algebra $\mathbb{C}\langle P, Q\rangle$ and arrived at the periodic orbits set of the $(2,1)$-De Bruijn graph. We showed that the reading of all the patterns of periodic orbits present at a vertex $k$ yielded all the monomials of $\mathscr{F}_{F}$ present at $k$ and whose contraction gave the polynomials in $P$ and $Q$. Let us now equip the periodic orbits with the coproduct of $\mathscr{E}$. Thanks to this coproduct, we will be able to speak of the growth of periodic orbits and to recover the quantum random walk over $\mathbb{Z}$ from their reading.

Definition 4.26 (growth of periodic orbit). The growth operator $\mathscr{G}: \mathbb{P} \mathbb{O} \rightarrow \mathbb{P} \mathbb{O}$ is defined by

$$
\mathscr{G}(\langle\omega\rangle)=\sum_{k=1}^{l(\langle\omega\rangle)}\left\langle{ }^{k} \delta(\omega)\right\rangle,
$$

where ${ }^{k} \delta=\mathrm{id} \otimes \cdots \otimes \underbrace{\Delta}_{k} \otimes \cdots \otimes \mathrm{id}$ and $l(\langle\omega\rangle)$ is the length of the pattern of the periodic $\operatorname{orbit}\langle\omega\rangle$. 
Remark 4.27. As $\Delta\left(x_{i j}\right):=\sum_{m=+1,-1} x_{i m} \otimes x_{m j}$, it is easy to see that $\varphi: \mathbb{P} \mathbb{O} \rightarrow \mathbb{P O}$. We take into account all the possible substitutions coming from the coassociative coproduct. Observe that $\sum_{k=1}^{l(\langle\omega\rangle)}\left\langle{ }^{k} \delta(\omega)\right\rangle$ can be decomposed into $\sum_{k=1}^{l(\langle\omega\rangle)} Y_{+1}^{k}$ and $\sum_{k=1}^{l(\langle\omega\rangle)} Y_{-1}^{k}$, where $Y_{ \pm 1}^{k} \in \mathbb{P} \mathbb{O}$ and where \pm 1 corresponds to the decomposition of the coproduct $\Delta$.

Remark 4.28. Observe also that the coproduct transforms a letter of index $k$ into two letters of index $k+1$ and $k-1$, that is, the coassociative coproduct leaves the index invariant. For instance, $\Delta(b)=a b+b d$ and $\operatorname{Ind}(b)=0 \mapsto(\operatorname{Ind}(a b)=-1)+(\operatorname{Ind}(b d)=+1)$.

THeOREM 4.29. The growth operator applied to all the periodic orbits at time $t=n$ will yield all the periodic orbits, perhaps with repetitions, at time $t=n+1$.

Proof. All the labels $i$ in $x_{i}$ will be understood modulo $n$, that is, $x_{i \bmod n}$. Let $\omega=x_{i_{1} i_{2}} x_{i_{2} i_{3}} \cdots$ $x_{i_{n-1} i_{n}}$ be a monomial of $\mathscr{F}$ present at time $t=n$, its completion yields the periodic orbit $\left\langle x_{i_{1} i_{2}} \cdots x_{i_{n-1} i_{n}} x_{i_{n} i_{1}}\right\rangle$ whose reading will give us the monomials $x_{i_{k} i_{k+1}} x_{i_{k+1} i_{k+2}} \cdots x_{i_{k+n-2} i_{k+n-1}}$, for $k=1, \ldots, N$. By definition of the quantum random walk, we have to multiply them by $P$ and $Q$ to have the new polynomials present at time $t=n+1$. Let see how it works on $\omega$ itself. We get $\mathscr{C}\left(x_{i_{1} i_{2}} x_{i_{2} i_{3}} \cdots x_{i_{n-1} i_{n}}\right) P$ and $\mathscr{C}\left(x_{i_{1} i_{2}} x_{i_{2} i_{3}} \cdots x_{i_{n-1} i_{n}}\right) Q$. These two polynomials come from the contraction of two monomials present at time $t=n+1, x_{i_{1} i_{2}} x_{i_{2} i_{3}} \cdots x_{i_{n-1} i_{n}}$ $x_{i_{n} i_{m}}$ and $x_{i_{1} i_{2}} x_{i_{2} i_{3}} \cdots x_{i_{n-1} i_{n}} x_{i_{n} i_{m^{\prime}}}$ with $m \neq m^{\prime}$. By completion of these two monomials, we get $\left\langle x_{i_{1} i_{2}} x_{i_{2} i_{3}} \cdots x_{i_{n-1} i_{n}} x_{i_{n} i_{m}} x_{i_{m} i_{1}}\right\rangle$ and $\left\langle x_{i_{1} i_{2}} x_{i_{2} i_{3}} \cdots x_{i_{n-1} i_{n}} x_{i_{n} i_{m^{\prime}}} x_{i_{m^{\prime}} i_{1}}\right\rangle$. The sum of these two periodic orbits is obviously equal to $\left\langle x_{i_{1} i_{2}} x_{i_{2} i_{3}} \cdots x_{i_{n-1} i_{n}} \Delta\left(x_{i_{n} i_{1}}\right)\right\rangle$. Now, fix $k$. By computing the labels modulo $n$, the contraction of the monomial $x_{i_{k} i_{k+1}} x_{i_{k+1} i_{k+2}} \cdots x_{i_{k+n-2} i_{k+n-1}}$ multiplied by $P$ and $Q$ comes from the contraction of the monomial $x_{i_{k} i_{k+1}} x_{i_{k+1} i_{k+2}} \cdots$ $x_{i_{k+n-2} i_{k+n-1}} x_{i_{k+n-1} i_{m}}$ and the monomial $x_{i_{k} i_{k+1}} x_{i_{k+1} i_{k+2}} \cdots x_{i_{k+n-2} i_{k+n-1}} x_{i_{k+n-1} i_{m^{\prime}}}$. The completion of these two monomials comes from the periodic orbit $\left\langle x_{i_{k} i_{k+1}} x_{i_{k+1} i_{k+2}} \cdots x_{i_{k+n-2} i_{k+n-1}} \Delta\left(x_{i_{k+n-1} i_{k}}\right)\right\rangle$. The last term $\Delta\left(x_{i_{k+n-1} i_{k}}\right)$ is equal to $\Delta\left(x_{i_{k-1} i_{k}}\right)$. That is why the growth operator works on periodic orbits to recover monomials at time $t=n+1$ from the periodic orbits at time $t=n$.

Remark 4.30. It is worth noticing that the Markovian coproduct is closely related to the coassociative coproduct. Indeed, a monomial $x_{i_{1} i_{2}} x_{i_{2} i_{3}} \cdots x_{i_{n-1} i_{n}}$ multiplied by $P$ and $Q$ will give the monomials $x_{i_{1} i_{2}} x_{i_{2} i_{3}} \cdots \Delta_{M}\left(x_{i_{n-1} i_{n}}\right)$, see Lemma 4.6, whereas its completion will use the coassociative coproduct. For instance, consider the monomial $a b$. The contraction of $a b$ multiplied by $P$ and $Q$ comes from the contraction of $a b c$ and $a b d$. Its completion will yield $a b c a$ and $a b d c$, which is $\langle a b \Delta(c)\rangle$. Therefore, $a b \rightarrow a b c+a b d=a \Delta_{M}(b) \stackrel{\text { Comp }}{\longrightarrow}$ $\langle a b \Delta(c)\rangle$.

4.1. Reconstruction of the quantum walk from periodic orbits of $\operatorname{Gr}(\mathscr{E})$. With the results we have obtained, we will now start with the set of periodic orbits of $\operatorname{Gr}(\mathscr{E})$ to recover the combinatorics of the quantum random walk. We now consider the space $\widehat{\mathbb{P O}}$, subvector space of $\mathscr{H} \otimes \mathbb{P O}:=\widehat{\mathbb{P O}}$, spanned by the vectors $|\operatorname{Ind}(\langle\omega\rangle)\rangle \otimes\langle\omega\rangle$, where $\langle\omega\rangle \in \mathbb{P O}$. The growth operator is now extended to $\widehat{\mathbb{P O}}$, that is, $\widehat{G}: \widehat{\mathbb{P O}} \rightarrow \widehat{\mathbb{P O}}$ and such that $\hat{\mathscr{G}}|\operatorname{Ind}(\langle\omega\rangle)\rangle \otimes\langle\omega\rangle:=\sum_{k=1}^{l(\langle\omega\rangle)}\left(\left|\operatorname{Ind}\left(Y_{+1}^{k}\right)\right\rangle \otimes Y_{+1}^{k}+\left|\operatorname{Ind}\left(Y_{-1}^{k}\right)\right\rangle \otimes Y_{-1}^{k}\right)$. Define, for all $n>1, \mathbb{P O} \mathbb{O}_{n}$ the set of all patterns of index $n$ and by convention $\mathbb{P O}_{2}:=\{\langle a a\rangle,\langle b c\rangle,\langle d d\rangle\}$. 
Denote $\Sigma_{n}^{\prime}:=\sum_{\langle\omega\rangle \in \mathbb{P} \mathbb{O}_{n-1}} \hat{\mathscr{G}}(|\operatorname{Ind}(\langle\omega\rangle)\rangle \otimes\langle\omega\rangle), n>2$. This sum is the sum of all patterns of periodic orbits, obtained perharps with repetition, present at time $n$. To avoid redundancy of information, define the (nonlinear) operator $J: \Sigma_{n}^{\prime}:=\sum \lambda|\operatorname{Ind}(\langle\omega\rangle)\rangle \otimes\langle\omega\rangle \mapsto$ $\sum|\operatorname{Ind}(\langle\omega\rangle)\rangle \otimes\langle\omega\rangle$, where $\lambda$ are integers, in such a way that a pattern $\langle\omega\rangle$, present in $\Sigma_{n}^{\prime}$ is present only one time in $J \Sigma_{n}^{\prime}$. Therefore, for all $n>1, J \Sigma_{n}^{\prime}$ is the sum of all patterns of periodic orbits, present at time $n$. Apply now the projector $|k\rangle\langle k| \otimes$ id on $J \Sigma_{n}^{\prime}$. Such a projector will yield all the orbits present at vertex $k$ and at time $n$. By reading them, all the monomials in $\mathscr{F}$ present at time $n$ at vertex $k$ will be obtained. By contraction, we will obtain all the polynomials at time $n$ at vertex $k$ generated by the quantum random walk.

At time $t=2$, we have two loops $\langle a a\rangle,\langle d d\rangle$, and $\langle b c\rangle$. Their readings yield $a, d$, and $b+c$. Their indexes are $-2,2$, and 0 . Their contractions yield $P P, Q Q$, and $P Q+$ $Q P$. At time $t=3$, the growth operator yields $\hat{\mathscr{G}}(|(-2)\rangle \otimes\langle a a\rangle) \mapsto 2|(-3)\rangle \otimes\langle a a a\rangle+$ $2|(-1)\rangle \otimes\langle b c a\rangle$ and $\hat{\mathscr{G}}(|(2)\rangle \otimes\langle d d\rangle) \mapsto 2|(3)\rangle \otimes\langle d d d\rangle+2|(1)\rangle \otimes\langle c b d\rangle$. Similarly, we obtain $\hat{\mathscr{G}}(|(0)\rangle \otimes\langle b c\rangle)$. By applying $J$ to $\Sigma_{3}^{\prime}$, we find $J \Sigma_{3}^{\prime}=|(-3)\rangle \otimes\langle a a a\rangle+|(-1)\rangle \otimes$ $\langle b c a\rangle+|(3)\rangle \otimes\langle d d d\rangle+|(1)\rangle \otimes\langle c b d\rangle$.

For instance, apply the projector $|-1\rangle\langle-1| \otimes$ id to $J \Sigma_{3}^{\prime}$. We find $|(-1)\rangle \otimes\langle b c a\rangle$. This pattern has to be present at time $t=3$ and at vertex -1 . Its reading yields $a b+b c+c a$ all of index -1 . Its contraction yields at time $t=3$ and at vertex -1 the polynomial $P P Q+$ $P Q P+Q P P$ as expected. By applying the growth operator at time $t=3$, we will still obtain all the orbits present at time $t=4$, and so forth.

\section{Relationships with classical systems}

We would like to establish a link between the quantum random walk we studied and their classical counterparts. For convenience, we recall in the following two subsections some results.

5.1. Classical random walk over $\mathbb{Z}$ and the Bernoulli shift. We consider the random walk over $\mathbb{Z}$, that is, we consider $\Omega=\{-1,1\}^{\mathbb{N}}$ equipped with the product measure $\mu^{\otimes \mathbb{N}}$, where $\mu=(1 / 2) \delta_{-1}+(1 / 2) \delta_{1}$. We consider the sequence of i.i.d. random variables $\left(X_{n}\right)_{n \in \mathbb{N}}$, with

$$
X_{n}: \Omega \longrightarrow\{-1,+1\}, \quad \text { such that } X_{n}(\omega)=\omega_{n}
$$

It is well known that this process and the symbolic dynamics generated by $x \mapsto 2 x \bmod 1$ are isomorphic. Indeed consider the i.i.d. process defined by, $Y_{n}: \Omega \rightarrow\{0,+1\}$, such that $Y_{n}(\omega)=\omega_{n}^{\prime}:=\left(\omega_{n}+1\right) / 2$. The set $\Omega=\{-1,1\}^{\mathbb{N}}$ becomes $\Omega^{\prime}=\{0,1\}^{\mathbb{N}}$ and $\mu$ becomes $\mu^{\prime}=(1 / 2) \delta_{0}+(1 / 2) \delta_{1}$. Consider the measurable function

$$
\Phi: \Omega^{\prime} \longrightarrow\left[0,1\left[, \quad \omega^{\prime} \longmapsto \sum_{n=0}^{\infty} \frac{\omega_{n}^{\prime}}{2^{n+1}}\right.\right.
$$

The cylinder $C=\left[Y_{0}=\omega_{0}^{\prime} ; \ldots ; Y_{l}=\omega_{l}^{\prime}\right]$, being the set of sequences starting by $\left(\omega_{0}^{\prime} ; \ldots ; \omega_{l}^{\prime}\right)$, will cover the interval $\left[\sum_{n=0}^{l}\left(\omega_{n}^{\prime} / 2^{n+1}\right) ; \sum_{n=0}^{l}\left(\omega_{n}^{\prime} / 2^{n+1}\right)+\sum_{n=l+1}^{\infty}\left(1 / 2^{n+1}\right)\right]$. We notice that 
$\operatorname{Leb}(C)=1 / 2^{l+1}$, where Leb is the Lebesgue measure. Let us consider the shift $\theta$ defined by $\left(\theta \omega^{\prime}\right)_{n}=\omega_{n+1}^{\prime}$. This shift leaves the Lebesgue measure of the cylinder $C$ invariant if we write $\theta\left(\left[Y_{0}=\omega_{0}^{\prime} ; \ldots ; Y_{l}=\omega_{l}^{\prime}\right]\right)=\left(\left[Y_{1}=\omega_{0}^{\prime} ; \ldots ; Y_{l+1}=\omega_{l}^{\prime}\right]\right)$. Moreover, we have $\Phi\left(\theta\left(\omega^{\prime}\right)\right)=2 \Phi\left(\omega^{\prime}\right) \bmod 1$.

The random walk over $\mathbb{Z}$ described by $\left(\Omega=\{-1,1\}^{\mathbb{N}}, \mu^{\otimes \mathbb{N}}, \theta\right)$ is isomorphic to $\left(\Omega^{\prime}=\right.$ $\left.\{0,1\}^{\mathbb{N}}, \mu^{\prime \otimes \mathbb{N}}, \theta^{\prime}\right)$ which is isomorphic to the chaotic system $([0,1[, \beta[0,1[$, Leb $, f: x \mapsto$ $2 x \bmod 1)$. As $\Phi \circ \theta^{\prime}=f \circ \Phi$, the diagram

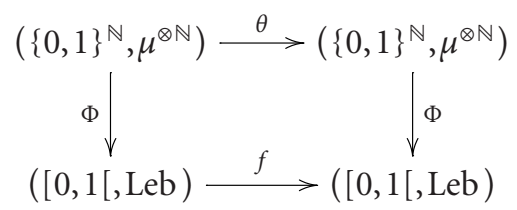

is commutative.

In [4], Biane proposes a noncommutative version of the Bernoulli process. Set $\Omega:=$ $\{+1,-1\}$, the probability space, and define the probability $\mathbb{P}(\{+1\})=p$ and $\mathbb{P}(\{-1\})=q$. The process $X: \Omega \rightarrow \mathbb{R}$ is defined as $X(+1)=+1$ and $X(-1)=-1$. By identifying $(1,0)$ with $(4 p)^{-1 / 2}(1+X)$ and $(0,1)$ with $(4 q)^{-1 / 2}(1-X)$, the space $L^{2}(\Omega, \mathbb{P})$ is isomorphic to $\mathbb{C}^{2}$. We notice that the algebra $L^{\infty}(\Omega, \mathbb{P})$, acting on $L^{2}$, can be identified with the algebra of diagonal matrices of $M_{2}(\mathbb{C})$. A natural noncommutative generalisation consists in lifting this commutative algebra into a noncommutative one, that is, $M_{2}(\mathbb{C})$. Notice that a subalgebra of $M_{2}(\mathbb{C})$ is also used in this framework.

5.2. Quantum graphs. Let $B$ be a bistochastic matrix representing a directed graph, that is, two vertices $x_{i}$ and $x_{j}$ are linked if and only if $B_{i j} \neq 0$. The matrix $B$ is said to be unistochastic if there exists a unitary matrix $U$ such that $B_{i j}=\left|U_{i j}\right|^{2}$. In this case, we say that the graph can be quantised. The notion of quantum graph was introduced as a toy model for studying quantum chaos by Kottos and Smilansky $[10,11]$. This notion was also studied by Tanner [17] and by Barra and Gaspard [2,3]. In this paper, we will follow another appraoch leading to quantum graphs put forward by Pakoński et al. [15] concerning onedimensional dynamical systems. They consider a one-dimensional mapping $f$ acting on $I=[0,1]$ such that $f: I \rightarrow I$ is piecewise linear and $f$ has to verify the following three conditions.

(1) There exists a Markov partition of the interval $I$ into $M$ equal cells $E_{i}:=[(i-$ $1) / M, i / M), i=1 \cdots M$, with $M$ a positive integer, and $f$ is linear on each cell $E_{i}$.

(2) For all $y \in I$,

$$
\sum_{x \in f^{-1}(y)} \frac{1}{f^{\prime}(x)}=1,
$$

where $f^{\prime}$ is the right derivative (defined almost everywhere on $I$ ).

(3) The finite transfer matrix $B$ describing the action of $f$ on the cells $E_{i}$ is unistochastic. 

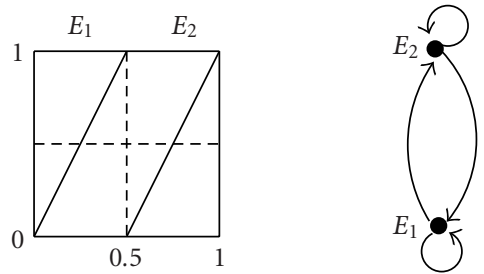

Figure 5.1

Remark 5.1. On each cell, $f$ concides with the function $f_{i}:[(i-1) / M, i / M) \rightarrow I, x \mapsto$ $c_{i} x+b_{i}$, where the $c_{i}$ have to be nonzero integers and the $b_{i}$ are rational. The unistochastic matrix $B$ is an $M$-by- $M$ matrix and $B_{i j}=1 /\left|c_{i}\right|$. Therefore, the probability of visiting the cell $E_{j}$ from $E_{i}$ is equal to $1 / f^{\prime}(x)$, with $x \in E_{i}$ and $f(x) \in E_{j}$.

Remark 5.2. The Kolmogorov-Sinai entropy of the Markov chain generated by the bistochastic matrix $B$ [7] is

$$
H_{\mathrm{KS}}=-\sum_{i=1}^{M} \tilde{p}_{i} \sum_{j=1}^{M} B_{i j} \log B_{i j}
$$

where $\tilde{p}$ is the normalised left eigenvector of $B$ such that $\tilde{p} B=\tilde{p}$, with $\sum_{i=1}^{M} \tilde{p}_{i}=1$. This equation gives the dynamical entropy of the system since the Markov partition on $M$ equal cells is a generating partition of the system. As the transition matrix is bistochastic, all the components of $\tilde{p}$ are equal to $1 / M$. Thus $H_{\mathrm{KS}}=0$ if and only if all the $B_{i j} \in\{0,1\}$. This entails that $\left|f^{\prime}(x)\right|=1$, that is, the system is regular. With the conditions stated above, the converse is true.

Example 5.3 (chaotic system, the Bernoulli shift). The Bernoulli shift is described by $f: x \mapsto 2 x \bmod 1$, where $x \in[0,1]$ (see Figure 5.1). This map is associated with the unistochastic matrix $B_{2}=(1 / 2)\left(\begin{array}{ll}1 & 1 \\ 1 & 1\end{array}\right)$.

We recall the following result.

Theorem 5.4 (see [15]). With the assumptions described in Section 5.2 on the piecewise linear map $f$, to every periodic orbit of period $n$ of the dynamical system described by $f$ corresponds a unique periodic orbit of period $n$ of the directed graph described by the associated unistochastic matrix, that is, by the (2,1)-De Bruijn graph.

We denote by $K$ such a bijection. That is, $K: \mathbb{P O} \rightarrow \mathbb{P O}[x \mapsto 2 x \bmod 1]$, where $\mathbb{P} \mathbb{O}[x \mapsto$ $2 x \bmod 1]$ denotes the $k$-vector space of patterns associated with the periodic orbits of the classical system $x \mapsto 2 x \bmod 1$.

Remark 5.5. Let $B_{2}$ be the unistochastic matrix associated with the chaotic map $x \mapsto$ $2 x \bmod 1$. One of the possible quantisation of this chaotic map is the so-called Hadamard matrix, $U_{\mathrm{H}}=(1 / \sqrt{2})\left(\begin{array}{cc}1 & 1 \\ 1 & -1\end{array}\right)$. 


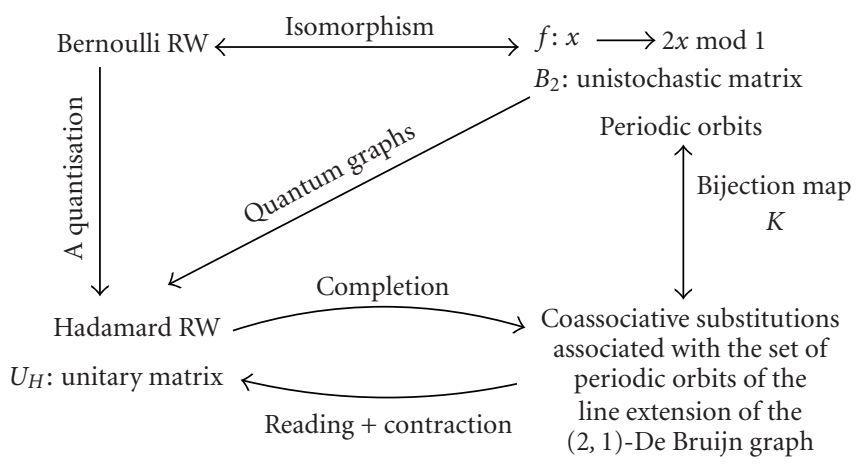

Figure 6.1

\section{Summary}

We now summarise our results in Figure 6.1.

\section{Appendix}

\section{A. Link with language theory}

In this appendix, we recall briefly the notion of grammar. We briefly show some points between grammar theory and the previous work.

Recall that a grammar with nonterminal symbols is a 4-tuple $G=\left(W, V, U, n_{0}\right)$, where $W$ is a finite set (its elements are called nonterminal symbols), $V$ is also a finite set such that $V \cap W=\varnothing$. The production set $U$ is a subset of pairs $u=(\alpha \rightarrow \beta)$, where $\alpha \in S=$ $W \cup V$ containing at least one symbol of $W$ and $\beta \in S^{*}$. The finite set $S$ plays the rôle of the alphabet and $S^{*}$ is the set of all finite strings over the alphabet $S$ including the empty one. The symbol $n_{0}$ belongs to $W$.

A grammar is called right linear if all the productions are of the form $n \rightarrow \alpha m$, where $n, m \in W$ and $\alpha \in V^{*}$. It is called context-free if all the productions are of the form $n \rightarrow \alpha$, where $n \in W$ and $\alpha \in V^{*}$. The language $L(G)$ generated by $G$ is the set of all sentences generated by $G$.

In this paper, we use implicitly grammar theory. Indeed, embed $S=W \cup V:=\{A, B$, $C, D\} \cup\{a, b, c, d\}$ into its free $k$-vector space. Consider the Fock space $F(G):=\bigoplus_{n} k S^{\otimes n}$. Define the concatenation product of two symbols of $S, \beta$, and $\alpha$, by $\beta \otimes \alpha$. The space $S^{*}$ is then the space of all the finite sentences $\beta_{1} \otimes \cdots \otimes \beta_{k}, \beta_{i} \in S$. The language $L(G)$ generated by $G$ is the $k$-vector space spanned by all the sentences generated by $G$. The element $n_{0}$ is then viewed as an element of $k W$. Now consider the production rules

$$
\begin{aligned}
& U_{M}(\mathscr{F}):=\{A \longmapsto a \otimes A, A \longmapsto a \otimes B, B \longmapsto b \otimes C, B \longmapsto b \otimes D, C \longmapsto c \otimes A, C \longmapsto c \otimes B, \\
& D \longmapsto d \otimes D, D \longmapsto d \otimes C\},
\end{aligned}
$$


and the so-called coassociative production rules

$$
\begin{aligned}
U_{c}(\mathscr{F}):=\{A \longmapsto a \otimes A, A \longmapsto b \otimes C, B \longmapsto b \otimes D, B \longmapsto a \otimes B, C \longmapsto c \otimes A, C \longmapsto d \otimes C, \\
D \longmapsto d \otimes D, D \longmapsto c \otimes B\} .
\end{aligned}
$$

Consider the nonterminal symbol START $n_{0}:=A+B+C+D$. Then, the language $L(G$, $\left.U_{M}(\mathscr{F}), n_{0}\right)$ generated by $n_{0}$, that is, all the sentences of $L(G)$ obtained by applying the production rules $U_{M}(\mathscr{F})$ to the start symbol $n_{0}:=A+B+C+D$, is the same as the language $L\left(G, U_{c}(\mathscr{F}), n_{0}\right)$ generated by $n_{0}$, see Proposition 4.6. In addition, these grammars are right linear. Observe also that the grammar constructed on the set of periodic orbits or patterns of the graph $\mathscr{F}$ is context free. The production rules are still given by the coassociative coproduct of $\mathscr{F}$,

$$
\begin{aligned}
U_{c}(\text { Per orbs }):=\{A & \longmapsto A \otimes A, A \longmapsto B \otimes C, B \longmapsto B \otimes D, B \longmapsto A \otimes B, C \longmapsto C \otimes A, \\
C & \longmapsto D \otimes C, D \longmapsto D \otimes D, D \longmapsto C \otimes B\} .
\end{aligned}
$$

The language of periodic orbits is the language generated by the sentence $A \otimes A+B \otimes C+$ $D \otimes D$.

\section{Acknowledgment}

The author wishes to thank Dimitri Petritis for useful discussions.

\section{References}

[1] A. Ambainis, E. Bach, A. Nayak, A. Vishwanath, and J. Watrous, One-dimensional quantum walks, Proceedings of the 33rd Annual ACM Symposium on Theory of Computing, ACM, New York, 2001, pp. 37-49.

[2] F. Barra and P. Gaspard, On the level spacing distribution in quantum graphs, J. Statist. Phys. 101 (2000), no. 1-2, 283-319.

[3] _ Transport and dynamics on open quantum graphs, Phys. Rev. E (3) 65 (2002), no. 1, part 2, 016205-016226.

[4] Ph. Biane, Marches de Bernoulli quantiques [Quantum Bernoulli walks], Séminaire de Probabilités, XXIV, 1988/1989, Lecture Notes in Math., vol. 1426, Springer, Berlin, 1990, pp. 329-344.

[5] V. F. R. Jones, Index for subfactors, Invent. Math. 72 (1983), no. 1, 1-25.

[6] S. A. Joni and G.-C. Rota, Coalgebras and bialgebras in combinatorics, Stud. Appl. Math. 61 (1979), no. 2, 93-139.

[7] A. Katok and B. Hasselblatt, Introduction to the Modern Theory of Dynamical Systems, Encyclopedia of Mathematics and Its Applications, vol. 54, Cambridge University Press, Cambridge, 1995.

[8] N. Konno, Quantum random walks in one dimension, Quantum Inf. Process. 1 (2002), no. 5, 345-354 (2003).

[9] N. Konno, T. Namiki, and T. Soshi, Symmetry of distribution for the one-dimensional Hadamard walk, Interdiscip. Inform. Sci. 10 (2004), no. 1, 11-22, The Proceedings of the Second Sendai Workshop on Quantum Probability and Quantum Information. 
[10] T. Kottos and U. Smilansky, Quantum chaos on graphs, Phys. Rev. Lett. 79 (1997), no. 24, 47944797.

[11] Periodic orbit theory and spectral statistics for quantum graphs, Ann. Physics 274 (1999), no. 1, 76-124.

[12] Ph. Leroux, An algebraic framework of weighted directed graphs, Int. J. Math. Math. Sci. 2003 (2003), no. 58, 3657-3678.

[13] Tiling the $\left(n^{2}, 1\right)$-De Bruijn graph with $n$ coassociative coalgebras, Comm. Algebra 32 (2004), no. 8, 2949-2967.

[14] From entangled codipterous coalgebras to coassociative manifolds, preprint, 2002, http://arxiv.org/abs/math.QA/0301080.

[15] P. Pakoński, K. Życzkowski, and M. Kuś, Classical 1D maps, quantum graphs and ensembles of unitary matrices, J. Phys. A 34 (2001), no. 43, 9303-9317.

[16] K. H. Rosen, J. G. Michaels, J. L. Gross, J. W. Grossman, and D. R. Shier (eds.), Handbook of Discrete and Combinatorial Mathematics, CRC Press, Florida, 2000.

[17] G. Tanner, Spectral statistics for unitary transfer matrices of binary graphs, J. Phys. A 33 (2000), no. 18, 3567-3585.

Philippe Leroux: Institut de Recherche Mathématique, Université de Rennes I and CNRS UMR 6625, Campus de Beaulieu, 35042 Rennes Cedex, France

E-mail address: philippe.leroux@univ-rennes1.fr 


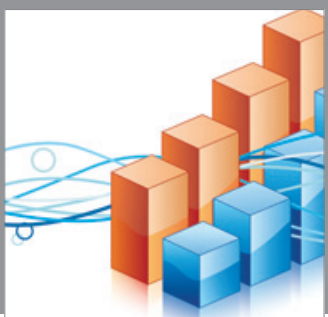

Advances in

Operations Research

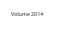

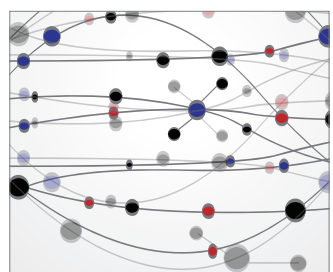

\section{The Scientific} World Journal
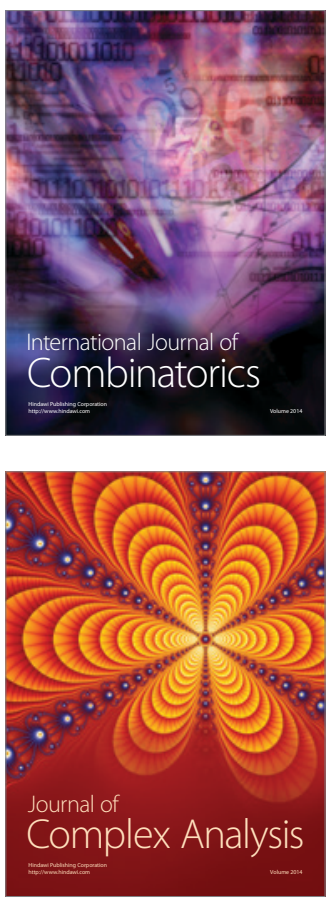

International Journal of

Mathematics and

Mathematical

Sciences
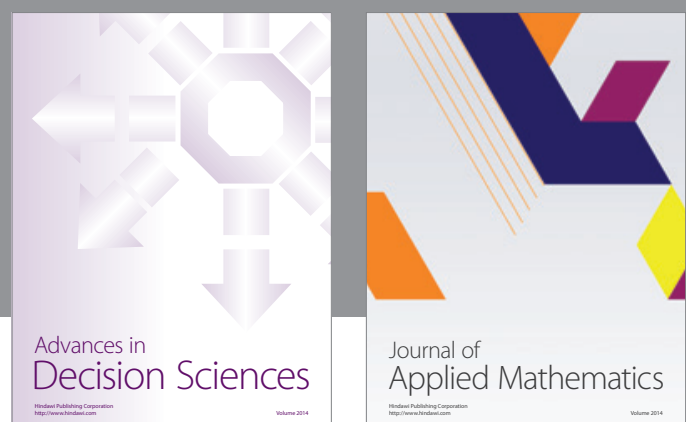

Journal of

Applied Mathematics
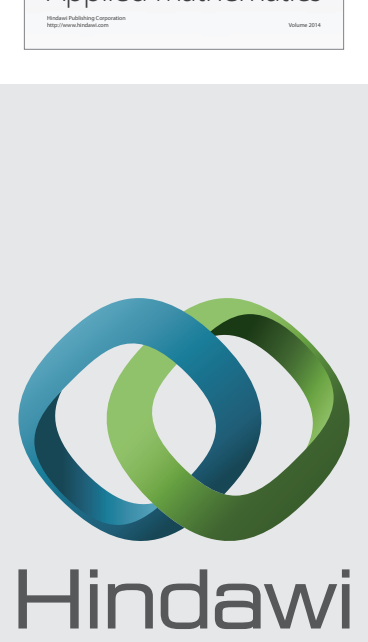

Submit your manuscripts at http://www.hindawi.com
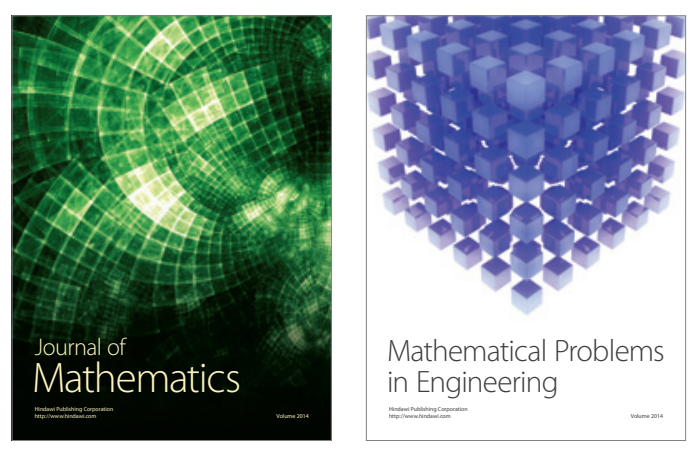

Mathematical Problems in Engineering
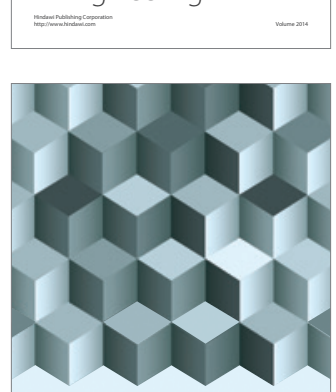

Journal of

Function Spaces
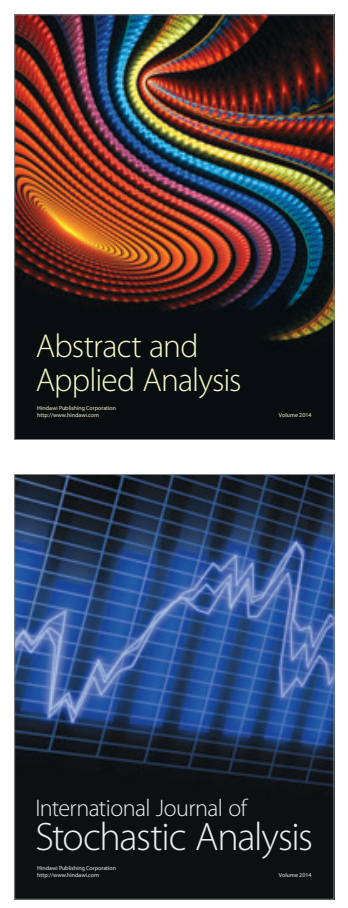

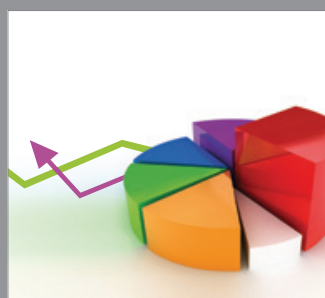

ournal of

Probability and Statistics

Promensencen
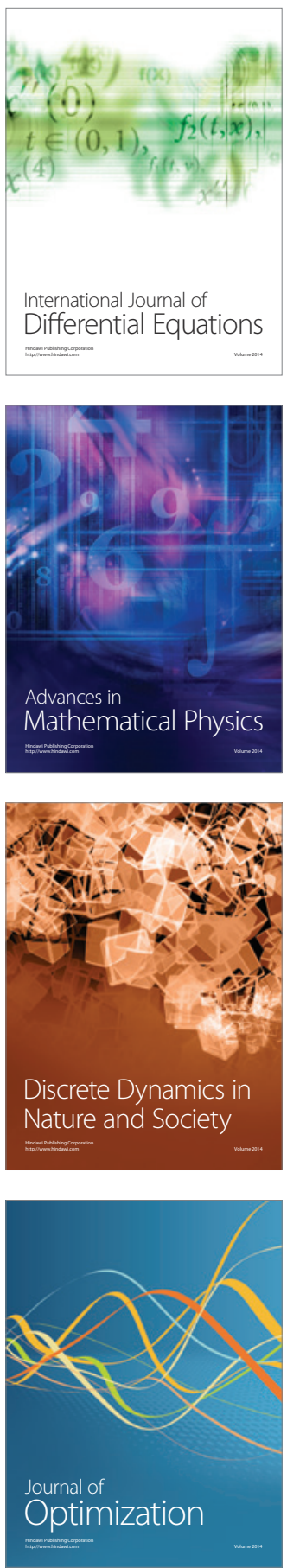